\title{
HIMPUNAN LEMBUT KABUR INTUISIONISTIK BERNILAI INTERVAL DAN SIFAT-SIFATNYA
}

\author{
SISRI WAHYUNI, NOVA NOLIZA BAKAR \\ Jurusan Matematika, \\ Fakultas Matematika dan Ilmu Pengetahuan Alam, Universitas Andalas, \\ Kampus UNAND Limau Manis Padang, Indonesia
}

\begin{abstract}
Abstrak. Sesuai perkembangan teori himpunan kabur, teori ini dapat dibedakan atas: teori himpunan lembut, himpunan lembut kabur, himpunan kabur intuisionistik, himpunan lembut kabur intuisionistik, himpunan kabur bernilai interval, himpunan lembut kabur bernilai interval, himpunan kabur intuisionistik bernilai interval, serta himpunan lembut kabur intuisionistik bernilai interval. Teori himpunan lembut kabur intuisionistik bernilai interval ini merupakan penggabungan dari teori himpunan kabur intuisionistik bernilai interval dengan himpunan lembut. Dalam himpunan lembut kabur intuisionistik bernilai interval ada beberapa operasi dan operator yang didefinisikan. Pada tulisan ini dibahas beberapa sifat dari himpunan lembut kabur intuisionistik bernilai interval yang terkait dengan operasi dan operator yang didefinisikan tersebut.
\end{abstract}

Kata Kunci: Himpunan kabur, himpunan lembut, himpunan lembut kabur, himpunan kabur intuisionistik, himpunan lembut kabur intuisionistik, himpunan kabur bernilai interval, himpunan lembut kabur bernilai interval, himpunan kabur intuisionistik bernilai interval, himpunan lembut kabur intuisionistik bernilai interval

\section{Pendahuluan}

Teori himpunan kabur $(F S)$ pertama kali diperkenalkan oleh Prof. L. A. Zadeh pada tahun 1965. Teori ini memiliki keterbatasan, sehingga Molodtsov (1999) [2] menginisiasi konsep baru yang disebut himpunan lembut (soft set/SS). Kemudian Maji dkk.(2001) [8] memperkenalkan konsep himpunan lembut kabur (fuzzy soft sets/FSS) yang merupakan kombinasi antara SS dan FS. Pada tahun (2009) [7] Maji dkk. mengkaji tentang himpunan lembut kabur intuisionistik (intuitionistic fuzzy soft set/IFSS) yang merupakan gabungan dari himpunan kabur intuisionistik (intuitionistic fuzzy set/IFS) dan himpunan lembut (soft set/SS).

Dalam beberapa kasus nyata, ditemukan bahwa derajat keanggotaan dari suatu elemen tidak selalu tunggal, tetapi merupakan himpunan dari beberapa nilai yang berbeda antara 0 dan 1 , sehingga Peneliti mendefinisikan $d k$ tersebut sebagai suatu interval dalam interval $[0,1]$. Kajian ini dinamakan dengan himpunan kabur bernilai interval (interval-valued fuzzy set/IVFS).

Kajian yang terkait dengan IVFS terus berkembang diantaranya himpunan lembut kabur bernilai interval (interval-valued fuzzy soft sets/IVFSS) yang diperkenalkan oleh Yang dkk.(2009) [12]. Pada tulisan ini dikaji kembali teori himpunan lembut kabur intuisionistik bernilai interval (interval-valued intuitionistic fuzzy soft set/IVIFSS) yang diperkenalkan oleh Jiang dkk. (2010). 


\section{Landasan Teori}

Definisi 2.1. [2] Misalkan $U$ adalah himpunan semesta, $P(U)$ adalah suatu himpunan kuasa atas $U$, dan $E$ adalah suatu himpunan parameter. Pasangan $\langle F, E\rangle$ disebut suatu himpunan lembut (soft set) atas $U$ jika dan hanya jika $F$ adalah pemetaan yang diberikan oleh $F: E \rightarrow P(U)$, yang dapat dinyatakan sebagai himpunan pasangan terurut yang berbentuk:

$$
\langle F, E\rangle=\{\langle\varepsilon, f(\varepsilon)\rangle \mid \varepsilon \in E, f(\varepsilon) \in P(U)\} .
$$

Definisi 2.2. [6] Himpunan kabur bernilai interval (interval-valued fuzzy sets/IVFS) $X$ atas himpunan semesta $U$, adalah pemetaan yang diberikan oleh $X: U \rightarrow \operatorname{int}[0 ; 1]$, dimana int $[0 ; 1]$ adalah himpunan dari seluruh subinterval tertutup dari interval [0;1]. Himpunan dari seluruh himpunan kabur bernilai interval dinotasikan dengan $\mathcal{I} \mathcal{V} \mathcal{F}(\mathcal{U})$.

Definisi 2.3. [12] Misalkan $U$ adalah himpunan semesta dan E adalah suatu himpunan parameter. Misalkan $A \subseteq E$. Pasangan $\langle F, A\rangle$ disebut himpunan lembut kabur bernilai interval (interval-valued fuzzy soft set/IVFSS) atas U, jika F adalah pemetaan yang diberikan oleh $F: A \rightarrow \mathcal{I} \mathcal{V} \mathcal{F}(\mathcal{U})$.

Definisi 2.4. [4] Suatu himpunan kabur intuisionistik bernilai interval (intervalvalued intuitionistic fuzzy set/IVIFS) A atas himpunan semesta X, adalah- suatu himpunan yang berbentuk

$$
A=\left\{\left\langle x, \mu_{A}(x), \gamma_{A}(x)\right\rangle \mid x \in X\right\},
$$

dimana $\mu_{A}: X \rightarrow \operatorname{int}[0 ; 1]$ dan $\gamma_{A}: X \rightarrow$ int $[0 ; 1]$ berturut-turut menyatakan derajat keanggotaan dan ketidakanggotaan dari $x \in X$ pada hmpunan $A$, dan int $[0 ; 1]$ adalah himpunan dari seluruh subinterval tertutup dari interval $[0 ; 1]$ yang memenuhi kondisi : $\forall x \in X$, sup $\mu_{A}(x)+\sup \gamma_{A}(x) \leq 1$. Himpunan dari seluruh IVIFS atas $X$ dinotasikan dengan $\mathcal{I} \mathcal{V} \mathcal{I} \mathcal{F}(\mathcal{X})$.

Untuk sebarang himpunan $A \subseteq[0 ; 1]$ didefinisikan $\underline{A}=\inf A \operatorname{dan} \bar{A}=\sup A$. Gabungan, irisan, serta komplemen dari himpunan kabur intuisionistik bernilai interval didefinisikan sebagai berikut.

Definisi 2.5. [4] Misalkan $A, B \in \mathcal{I} \mathcal{I} \mathcal{I} \mathcal{F}(\mathcal{X})$, maka

(1) Gabungan dari $A$ dan $B$, dinotasikan dengan $A \cup B$ dimana

$A \cup B=\left\{\left\langle x,\left[\sup \left(\underline{\mu}_{A}(x), \underline{\mu}_{B}(x)\right), \sup \left(\bar{\mu}_{A}(x), \bar{\mu}_{B}(x)\right)\right],\left[\inf \left(\underline{\gamma}_{A}(x), \underline{\gamma}_{B}(x)\right), \inf \left(\bar{\gamma}_{A}(x), \bar{\gamma}_{B}(x)\right)\right]\right\rangle ; x \in X\right\}$

(2) Irisan dari $A$ dan $B$ dinotasikan dengan $A \cap B$ dimana

$A \cap B=\left\{\left\langle x,\left[\inf \left(\underline{\mu}_{A}(x), \underline{\mu}_{B}(x)\right), \inf \left(\bar{\mu}_{A}(x), \bar{\mu}_{B}(x)\right)\right],\left[\sup \left(\underline{\gamma}_{A}(x), \underline{\gamma}_{B}(x)\right), \sup \left(\bar{\gamma}_{A}(x), \bar{\gamma}_{B}(x)\right)\right]\right\rangle ; x \in X\right\}$.

(3) Komplemen dari $A$ dinotasikan dengan $A^{c}$ dimana

$$
A^{c}=\left\{\left\langle x, v_{A}(x), \mu_{A}(x) ; x \in X\right\} .\right.
$$




\section{Pembahasan}

Berikut akan dijelaskan tentang himpunan lembut kabur intuisionistik bernilai interval, yang merupakan lanjutan dari teori himpunan lembut kabur intuisionistik atau teori himpunan lembut kabur bernilai interval.

\subsection{Himpunan Lembut Kabur Intuisionistik Bernilai Interval (Interval-Valued Intuisionistik Fuzzy Soft Set)}

Definisi 3.1. [11] Misalkan U himpunan semesta dan E suatu himpunan parameter. Suatu pasangan $\langle F, A\rangle$ dikatakan himpunan lembut kabur intuisionistik bernilai interval atas $U$, jika $F$ adalah suatu pemetaan yang diberikan oleh $F$ : $A \rightarrow \mathcal{I} \mathcal{I} \mathcal{F}(\mathcal{U})$, dimana $\mathcal{I} \mathcal{V} \mathcal{F}(\mathcal{U})$ adalah himpunan dari seluruh himpunan kabur intuisionistik bernilai interval atas $U$, dan $A \subseteq E$. Himpunan $\langle F, A\rangle$ dapat ditulis sebagai

$$
\langle F, A\rangle=\left\{\left\langle e_{i}, F\left(e_{i}\right)\right\rangle \mid F\left(e_{i}\right) \in \mathcal{I} \mathcal{V} \mathcal{I} \mathcal{F}(\mathcal{U}), e_{i} \in A\right\},
$$

dengan $F\left(e_{i}\right)=\left\{\left\langle x, \mu_{F\left(e_{i}\right)}(x), \gamma_{F\left(e_{i}\right)}(x)\right\rangle \mid x \in U, e_{i} \in A\right\}$, dan $\mu_{F\left(e_{i}\right)}(x), \gamma_{F\left(e_{i}\right)}(x)$ berturut-turut menyatakan derajat keanggotaan dan derajat ketidakanggotaan dari $x \in U$ pada himpunan $\langle F, A\rangle$.

Untuk sebarang parameter $\varepsilon \in A, F(\varepsilon)$ adalah nilai dari parameter $\epsilon$ yang merupakan suatu himpunan kabur intuisionistik bernilai interval atas U dimana $x$ $\in U$ dan $\varepsilon \in A$.

Definisi 3.2. [11] Misalkan $U$ himpunan semesta, E himpunan parameter, $A \subseteq$ $E$ dan $B \subseteq E$. Himpunan $\langle F, A\rangle$ dan $\langle G, B\rangle$ adalah dua himpunan lembut kabur intuisionistik bernilai interval. Himpunan $\langle F, A\rangle$ disebut himpunan bagian lembut kabur intuisionistik bernilai interval $\langle G, B\rangle$ jika dan hanya jika

(1) $A \subseteq B$;

(2) $\forall \epsilon \in A, F(\epsilon)$ adalah himpunan bagian kabur intuisionistik bernilai interval $G(\epsilon)$, dimana untuk setiap $x \in U$ dan $\epsilon \in A, \underline{\mu}_{F(\epsilon)}(x) \leq \underline{\mu}_{G(\epsilon)}(x), \bar{\mu}_{F(\epsilon)}(x) \leq$ $\bar{\mu}_{G(\epsilon)}(x), \underline{\gamma}_{F(\epsilon)}(x) \geq \underline{\gamma}_{G(\epsilon)}(x), \operatorname{dan} \bar{\gamma}_{F(\epsilon)}(x) \geq \bar{\gamma}_{G(\epsilon)}(x)$.

Hubungan antara $\langle F, A\rangle$ dan $\langle G, B\rangle$ diatas dinotasikan dengan $\langle F, A\rangle \Subset\langle G, B\rangle$.

Definisi 3.3. [11] Misalkan $\langle F, A\rangle$ dan $\langle G, B\rangle$ adalah dua himpunan lembut kabur intuisionistik bernilai interval atas $U,\langle F, A\rangle$ dan $\langle G, B\rangle$ dikatakan sama jika dan hanya jika

(1) $\langle F, A\rangle$ adalah himpunan bagian lembut kabur intuisionistik bernilai interval $\langle G, B\rangle$, atau $\langle F, A\rangle \Subset\langle G, B\rangle$.

(2) $\langle G, B\rangle$ adalah himpunan bagian lembut kabur intuisionistik bernilai interval $\langle F, A\rangle$, atau $\langle G, B\rangle \Subset\langle F, A\rangle$.

Hubungan antara $\langle F, A\rangle$ dan $\langle G, B\rangle$ dinotasikan dengan $\langle F, A\rangle=\langle G, B\rangle$. 


\subsection{Operasi-Operasi dan Sifat-Sifat Himpunan Lembut Kabur Intuisionistik Bernilai Interval}

Berikut akan dijelaskan definisi beberapa operasi pada himpunan lembut kabur intuisionistik bernilai interval.

Definisi 3.4. [11] Misalkan $E=\left\{e_{1}, e_{2}, \cdots, e_{n}\right\}$ himpunan parameter. Bukan himpunan $E$ yang dinotasikan dengan $\rceil E$, didefinisikan sebagai

$$
\rceil E=\left\{\neg e_{1}, \neg e_{2}, \cdots, \neg e_{n}\right\},
$$

dimana $\neg e_{i}=$ bukan $e_{i}$.

Definisi 3.5. [11] Komplemen dari himpunan lembut kabur intuisionistik bernilai interval $\langle F, A\rangle$ yang dinotasikan dengan $\langle F, A\rangle^{C}$, didefinisikan sebagai

$$
\left.\langle F, A\rangle^{C}=\left\langle F^{C},\right\rceil A\right\rangle,
$$

dimana $\left.F^{C}:\right\rceil A \rightarrow \mathcal{I} \mathcal{I} \mathcal{F}(\mathcal{U})$ suatu pemetaan yang diberikan oleh

$$
F^{C}(\epsilon)=\left\langle x, \gamma_{F(\neg \epsilon)}(x), \mu_{F(\neg \epsilon)(x)}\right\rangle,
$$

dengan

$$
\left.\mu_{F(\neg \epsilon)}(x)=\gamma_{F(\epsilon)}(x) \gamma_{F(\neg \epsilon)}(x)=\mu_{F(\epsilon)}(x) \text { untuk semua } x \in U \text { dan } \epsilon \in\right\rceil A .
$$

Definisi 3.6. [11] Himpunan lembut kabur intuisionistik bernilai interval $\langle F, A\rangle$ atas $U$ dikatakan himpunan lembut kabur intuisionistik bernilai interval null jika $\forall \epsilon \in A, \mu_{F(\epsilon)}(x)=[0 ; 0], \gamma_{F(\epsilon)}(x)=[1 ; 1], \forall x \in U$, dan dinotasikan dengan $\Phi$.

Definisi 3.7. [11] Himpunan lembut kabur intuisionistik bernilai interval $\langle F, A\rangle$ atas $U$ dikatakan himpunan lembut kabur intuisionistik bernilai interval mutlak jika $\forall \epsilon \in A, \mu_{F(\epsilon)}(x)=[1 ; 1], \gamma_{F(\epsilon)}(x)=[0 ; 0], \forall x \in U$, dan dinotasikan dengan $\Sigma$.

Definisi 3.8. [11] Jika $\langle F, A\rangle$ dan $\langle G, B\rangle$ adalah dua himpunan lembut kabur intuisionistik bernilai interval atas $U, \operatorname{maka}\langle F, A\rangle D A N\langle G, B\rangle$ adalah suatu himpunan lembut kabur intuisionistik bernilai interval yang dinotasikan dengan $\langle F, A\rangle \wedge\langle G, B\rangle$, dan didefinisikan sebagai

$$
\langle F, A\rangle \wedge\langle G, B\rangle=\langle H, A \times B\rangle,
$$

dimana

$$
\begin{aligned}
H(\alpha, \beta)= & F(\alpha) \cap G(\beta), \forall(\alpha, \beta) \in A \times B, \\
H(\alpha, \beta)(x)= & \left\langle\inf \left(\underline{\mu}_{F(\alpha)}(x), \underline{\mu}_{G(\beta)}(x)\right), \inf \left(\bar{\mu}_{F(\alpha)}(x), \bar{\mu}_{G(\beta)}(x)\right), \quad\left[\sup \left(\underline{\gamma}_{F(\alpha)}(x), \underline{\gamma}_{G(\beta)}(x)\right),\right.\right. \\
& \left.\left.\quad \sup \left(\bar{\gamma}_{F(\alpha)}(x), \bar{\gamma}_{G(\beta)}(x)\right)\right]\right\rangle, \forall(\alpha, \beta) \in A \times B, x \in U .
\end{aligned}
$$

Definisi 3.9. [11] Jika $\langle F, A\rangle$ dan $\langle G, B\rangle$ adalah dua himpunan lembut kabur intuisionistik bernilai interval atas $U$, maka $\langle F, A\rangle$ ATAU $\langle G, B\rangle$ adalah suatu himpunan lembut kabur intuisionistik bernilai interval yang dinotasikan dengan $\langle F, A\rangle \vee\langle G, B\rangle$, dan didefinisikan sebagai

$$
\langle F, A\rangle \vee\langle G, B\rangle=\langle O, A \times B\rangle
$$


dimana

$$
\begin{aligned}
O(\alpha, \beta)= & F(\alpha) \cup G(\beta), \forall(\alpha, \beta) \in A \times B, \\
O(\alpha, \beta)(x)= & \left\langle\left[\sup \left(\underline{\mu}_{F(\alpha)}(x), \underline{\mu}_{G(\beta)}(x)\right), \sup \left(\bar{\mu}_{F(\alpha)}(x), \bar{\mu}_{G(\beta)}(x)\right], \quad \inf \underline{\gamma}_{F(\alpha)}(x), \underline{\gamma}_{G(\beta)}(x)\right),\right. \\
& \left.\left.\left(\bar{\gamma}_{F(\alpha)}(x), \bar{\gamma}_{G(\beta)}(x)\right)\right]\right\rangle, \forall(\alpha, \beta) \in A \times B, x \in U .
\end{aligned}
$$

Berikut teorema-teorema yang terkait dalam operasi pada IVIFS.

Teorema 3.10. [11] Misalkan $\langle F, A\rangle$ dan $\langle G, B\rangle$ adalah dua himpunan lembut kabur intuisionistik bernilai interval, maka sifat-sifat berikut berlaku:

(1) $(\langle F, A\rangle \wedge\langle G, B\rangle)^{C}=\langle F, A\rangle^{C} \vee\langle G, B\rangle^{C}$,

(2) $(\langle F, A\rangle \vee\langle G, B\rangle)^{C}=\langle F, A\rangle^{C} \wedge\langle G, B\rangle^{C}$.

Teorema 3.11. [11] Misalkan $\langle F, A\rangle,\langle G, B\rangle$, dan $\langle H, C\rangle$ adalah himpunan lembut kabur intuisionistik bernilai interval, maka sifat-sifat berikut berlaku:

(1) $\langle F, A\rangle \wedge(\langle G, B\rangle \wedge\langle H, C\rangle)=(\langle F, A\rangle \wedge\langle G, B\rangle) \wedge\langle H, C\rangle$,

(2) $\langle F, A\rangle \vee(\langle G, B\rangle \vee\langle H, C\rangle)=(\langle F, A\rangle \vee\langle G, B\rangle) \vee\langle H, C\rangle$.

Definisi 3.12. [11] Gabungan dari dua himpunan lembut kabur intuisionistik bernilai interval $\langle F, A\rangle$ dan $\langle G, B\rangle$ atas $U$, adalah himpunan lembut kabur intuisonistik bernilai interval $\langle H, C\rangle$ dimana $C=A \cup B$, dan $\forall \varepsilon \in C$, berlaku:

$\mu_{H(\varepsilon)}(x)= \begin{cases}\mu_{F(\varepsilon)}(x), & \text { jika } \varepsilon \in A-B, x \in U . \\ \mu_{G(\varepsilon)}(x), & \text { jika } \varepsilon \in B-A, x \in U . \\ {\left[\sup \left(\underline{\mu}_{F(\varepsilon)}(x), \underline{\mu}_{G(\varepsilon)}(x)\right), \sup \left(\bar{\mu}_{F(\varepsilon)}(x), \bar{\mu}_{G(\varepsilon)}(x)\right)\right],} & \text { jika } \varepsilon \in A \cap B, x \in U .\end{cases}$

$\gamma_{H(\varepsilon)}(x)= \begin{cases}\gamma_{F(\varepsilon)}(x), & \text { jika } \varepsilon \in A-B, x \in U . \\ \gamma_{G(\varepsilon)}(x), & \text { jika } \varepsilon \in B-A, x \in U . \\ {\left[\inf \left(\underline{\gamma}_{F(\varepsilon)}(x), \underline{\gamma}_{G(\varepsilon)}(x)\right), \inf \left(\bar{\gamma}_{F(\varepsilon)}(x), \bar{\gamma}_{G(\varepsilon)}(x)\right)\right],} & \text { jika } \varepsilon \in A \cap B, x \in U .\end{cases}$

dengan $\mu_{H(\varepsilon)}(x), \gamma_{H(\varepsilon)}(x)$ berturut-turut menyatakan derajat keanggotaan dan derajat ketidakanggotaan dari $x \in X$ pada himpunan $\langle H, C\rangle$.

Hubungan ini dinotasikan dengan $\langle F, A\rangle \cup\langle G, B\rangle=\langle H, C\rangle$.

Definisi 3.13. [11] Irisan dari dua himpunan lembut kabur intuisionistik bernilai interval $\langle F, A\rangle$ dan $\langle G, B\rangle$ atas $U$, adalah himpunan lembut kabur intuisonistik bernilai interval $\langle H, C\rangle$ dimana $C=A \cup B$, dan $\forall \varepsilon \in C$, berlaku:

$$
\begin{array}{r}
\mu_{H(\varepsilon)}(x)=\left\{\begin{array}{lr}
\mu_{F(\varepsilon)}(x), & \text { jika } \varepsilon \in A-B, x \in U . \\
\mu_{G(\varepsilon)}(x), & \text { jika } \varepsilon \in B-A, x \in U . \\
{\left[\inf \left(\underline{\mu}_{F(\varepsilon)}(x), \underline{\mu}_{G(\varepsilon)}(x)\right), \inf \left(\bar{\mu}_{F(\varepsilon)}(x), \bar{\mu}_{G(\varepsilon)}(x)\right)\right],} & \text { jika } \varepsilon \in A \cap B, x \in U .
\end{array}\right. \\
\gamma_{H(\varepsilon)}(x)= \begin{cases}\gamma_{F(\varepsilon)}(x), & \text { jika } \varepsilon \in A-B, x \in U . \\
\gamma_{G(\varepsilon)}(x), & \text { jika } \varepsilon \in B-A, x \in U . \\
{\left[\sup \left(\underline{\gamma}_{F(\varepsilon)}(x), \underline{\gamma}_{G(\varepsilon)}(x)\right), \sup \left(\bar{\gamma}_{F(\varepsilon)}(x), \bar{\gamma}_{G(\varepsilon)}(x)\right)\right],} & \text { jika } \varepsilon \in A \cap B, x \in U .\end{cases}
\end{array}
$$

dengan $\mu_{H(\varepsilon)}(x), \gamma_{H(\varepsilon)}(x)$ berturut-turut menyatakan derajat keanggotaan dan derajat ketidakanggotaan dari $x \in X$ pada himpunan $\langle H, C\rangle$.

Hubungan ini dinotasikan dengan $\langle F, A\rangle \cap\langle G, B\rangle=\langle H, C\rangle$.

Teorema 3.14. [11] Misalkan E suatu himpunan parameter, $A \subseteq$ E. Jika $\Phi$ adalah himpunan lembut kabur intuisionistik bernilai interval null, $\Sigma$ adalah himpunan 
lembut kabur intuisionistik bernilai interval mutlak, serta $\langle F, A\rangle$ dan $\langle F, E\rangle$ adalah dua himpunan lembut kabur intuisionistik bernilai interval, maka sifat-sifat berikut berlaku:

(1) $\langle F, A\rangle \uplus\langle F, A\rangle=\langle F, A\rangle$,

(2) $\langle F, A\rangle$ ก $\langle F, A\rangle=\langle F, A\rangle$,

(3) $\langle F, E\rangle \uplus \Phi=\langle F, E\rangle$,

(4) $\langle F, E\rangle \cap \Phi=\Phi$,

(5) $\langle F, E\rangle \in \Sigma=\Sigma$,

(6) $\langle F, E\rangle \cap \Sigma=\langle F, E\rangle$.

Teorema 3.15. [11] Jika $\langle F, A\rangle$ dan $\langle G, B\rangle$ adalah dua himpunan lembut kabur intuisionistik bernilai interval atas $U$, maka sifat-sifat berikut berlaku:

(1) $(\langle F, A\rangle \uplus\langle G, B\rangle)^{C}=\langle F, A\rangle^{C}$ ก $\langle G, B\rangle^{C}$,

(2) $(\langle F, A\rangle \text { ก }\langle G, B\rangle)^{C}=\langle F, A\rangle^{C}$ 巴 $\langle G, B\rangle^{C}$.

Teorema 3.16. [11] Misalkan $\langle F, A\rangle,\langle G, B\rangle$ dan $\langle H, C\rangle$ adalah tiga himpunan lembut kabur intuisionistik bernilai interval atas $U$, maka sifat-sifat berikut berlaku:

(1) $\langle F, A\rangle$ ก $(\langle G, B\rangle$ ก $\langle H, C\rangle)=(\langle F, A\rangle \cap\langle G, B)\rangle$ ก $\langle H, C\rangle$,

(2) $\langle F, A\rangle \uplus(\langle G, B\rangle \uplus\langle H, C\rangle)=(\langle F, A\rangle \uplus\langle G, B\rangle) \uplus\langle H, C\rangle$.

Definisi 3.17. [11] Operasi necessity pada himpunan lembut kabur intuisionistik bernilai interval $\langle F, A\rangle$, dinotasikan dengan $\square\langle F, A\rangle$, didefinisikan sebagai

$$
\square\langle F, A\rangle=\left\{\left\langle x, \mu_{\square F(\varepsilon)}(x), \gamma_{\square F(\varepsilon)}(x)\right\rangle \mid x \in U, \varepsilon \in A\right\},
$$

dimana

$$
\mu_{\square F(\varepsilon)}(x)=\left[\underline{\mu}_{F(\varepsilon)}(x), \bar{\mu}_{F(\varepsilon)}(x)\right] \text {, dan } \gamma_{\square F(\varepsilon)}(x)=\left[1-\bar{\mu}_{F(\varepsilon)}(x), 1-\underline{\mu}_{F(\varepsilon)}(x)\right] .
$$

Teorema 3.18. [11] Misalkan $\langle F, A\rangle$ dan $\langle G, B\rangle$ adalah dua himpunan lembut kabur intuisionistik bernilai interval atas $U$, maka sifat-sifat berikut berlaku:

(1) $\square(\langle F, A\rangle \uplus\langle G, B\rangle)=\square\langle F, A\rangle \uplus \square\langle G, B\rangle$,

(2) $\square(\langle F, A\rangle$ ก $\langle G, B\rangle)=\square\langle F, A\rangle$ ก $\square\langle G, B\rangle$,

(3) $\square \square(\langle F, A\rangle=\square\langle F, A\rangle$.

Definisi 3.19. [11] Operasi possibility pada himpunan lembut kabur intui-sionistik bernilai interval $\langle F, A\rangle$, dinotasikan dengan $\diamond\langle F, A\rangle$, didefinisikan sebagai

$$
\diamond\langle F, A\rangle=\left\{\left\langle x, \mu_{\diamond F(\varepsilon)}(x), \gamma_{\diamond F(\varepsilon)}(x)\right\rangle \mid x \in U, \varepsilon \in A\right\},
$$

dimana $\mu_{\diamond F(\varepsilon)}(x)=\left[1-\bar{\gamma}_{F(\varepsilon)}(x), 1-\underline{\gamma}_{F(\varepsilon)}(x)\right]$, dan $\gamma_{\diamond F(\varepsilon)}(x)=$ $\left[\underline{\gamma}_{F(\varepsilon)}(x), \bar{\gamma}_{F(\varepsilon)}(x)\right]$.

Teorema 3.20. [11] Misalkan $\langle F, A\rangle$ dan $\langle G, B\rangle$ adalah dua himpunan lembut kabur intuisionistik bernilai interval atas $U$, maka sifat-sifat berikut berlaku:

(1) $\diamond(\langle F, A\rangle \uplus\langle G, B\rangle)=\diamond\langle F, A\rangle \uplus \diamond\langle G, B\rangle$,

(2) $\diamond(\langle F, A\rangle$ ก $\langle G, B\rangle)=\diamond\langle F, A\rangle$ ก $\diamond\langle G, B\rangle$, 
(3) $\diamond \diamond(\langle F, A\rangle=\diamond\langle F, A\rangle$.

Teorema 3.21. [11] Misalkan $\langle F, A\rangle$ adalah himpunan lembut kabur intuisionistik atas $U$, maka sifat-sifat berikut berlaku:

(1) $\square\langle F, A\rangle \Subset\langle F, A\rangle \Subset \diamond\langle F, A\rangle$,

(2) $\diamond \square\langle F, A\rangle=\square\langle F, A\rangle$,

(3) $\square \diamond\langle F, A\rangle=\diamond\langle F, A\rangle$,

Teorema 3.22. [11] Misalkan $\langle F, A\rangle$ dan $\langle G, B\rangle$ adalah dua himpunan lembut kabur intuisionistik bernilai interval atas $U$, maka sifat-sifat berikut berlaku:

(1) $\square(\langle F, A\rangle \wedge\langle G, B\rangle)=\square\langle F, A\rangle \wedge \square\langle G, B\rangle$,

(2) $\square(\langle F, A\rangle \vee\langle G, B\rangle)=\square\langle F, A\rangle \vee \square\langle G, B\rangle$,

(3) $\diamond(\langle F, A\rangle \wedge\langle G, B\rangle)=\diamond\langle F, A\rangle \wedge \diamond\langle G, B\rangle$,

(4) $\diamond(\langle F, A\rangle \vee\langle G, B\rangle)=\diamond\langle F, A\rangle \vee \diamond\langle G, B\rangle$.

\section{Kesimpulan}

Himpunan lembut kabur intuisionistik bernilai interval merupakan penggabungan dari himpunan lembut dengan himpunan kabur intuisionistik bernilai interval. Dari pendefinisian operasi biner gabungan (ש), irisan ( $)$, DAN $(\wedge)$, serta ATAU $(\vee)$ pada himpunan lembut kabur intuisionistik bernilai interval diperoleh sifat asosiati dan distributif. Keempat operasi biner ini tetap dipertahankan dengan menggunakan operator necessity, possibility, serta necessity dan possibility.

\section{Ucapan Terima kasih}

Penulis mengucapkan terima kasih kepada Bapak Muhafzan, Ibu Monika Rianti Helmi, dan ibu Ferra Yanuar yang telah memberikan masukan dan saran sehingga makalah ini dapat diselesaikan dengan baik.

\section{Daftar Pustaka}

[1] Bartle, R.G. and Sherbert,D.R. 2000. Introduction to Real Analysis, Second Edition. Urbana-Champaign: University of Illinois.

[2] Molodtsov, D. 1999. Soft Set Theory. American Mathematical Society. 37: 19 $-31$

[3] Atanassov, K. 1986. Intuitionistic Fuzzy Sets. Fuzzy Sets and Systems. 20: 87 $-96$

[4] Atanassov, K., G. Gargov. 1989. Interval-Valued Intuitionistic Fuzzy Sets. Fuzzy Sets and Systems. 31: 343 - 349

[5] Zadeh, L.A. 1965. Fuzzy Sets. Information and Control. 8: 338 - 356

[6] Gorzalczany, M.B. 1987. A Method of Inference in Approximate Reasoning Based on Interval valued Fuzzy Sets. Fuzzy Sets and System. 21: $1-17$

[7] Maji, P.K., 2009. More on Intuitionistic Fuzzy Soft Sets. Lecture Notes in Computer Science. 5908: 231 - 240

[8] Maji, P.K., R. Biswas, A.R. Roy. 2001. Fuzzy Soft Sets. Fuzzy Mathematics. 9: $589-602$ 
Himpunan Lembut Kabur Intuisionistik Bernilai Interval 203

[9] Maji, P.K., R. Biswas, A.R. Roy. 2001. Intuitionistic Fuzzy Soft Sets. Fuzzy Mathematics. 9: 677 - 692

[10] Maji, P.K., R. Biswas, A.R. Roy. 2002. An Application of Soft Sets in A Decision Making Problem. Computers and Mathematics with Applications. 44: $1077-1083$

[11] Jiang Y. 2010. Interval-Valued Intuitionistic Fuzzy Soft Sets and Their Properties. Computers and Mathematics with Applications. 60: 906 - 918

[12] Yang, X.B. T.Y. Lin, J.Y. Yang, D.Yu. 2009. Combination of Interval-Valued Fuzzy Set and Soft Set. Computers and Mathematics with Applications. 58: $521-527$ 